\section{Neue ESC-Leitlinien zum Management bei Dyslipidämie}

\author{
Auch in der neuen Fassung der Leitlinie zur Therapie der Dyslipidämie \\ wird am Konzept einer an Zielwerten orientierten Senkung des LDL-Cho- \\ lesterins in Abhängigkeit vom individuellen Risiko festgehalten.
}

D ie europäische Kardiologie-Gesellschaft (ESC) und die europäische Atherosklerose-Gesellschaft (EAS) haben ihre Leitlinien zum Management bei Störungen des Lipidstoffwechsels auf den neuesten Stand gebracht. Die neuen Leitlinien sind Ende August beim ESC-Kongress 2016 in Rom vorgestellt und im „European Heart Journal" publiziert worden.

\section{Bestimmung des Risikos}

Da atherosklerotische Gefäßerkrankungen in aller Regel das Produkt vieler interagierender Risikofaktoren sind, macht die Bestimmung des individuellen kardiovaskulären Risikos eines Patienten den Anfang. Von der Höhe des ermittelten Risikos hängt die Intensität der zu ergreifenden präventiven Maßnahmen einschließlich Lipidsenkung ab.

Zur Bestimmung des Risikos werden heute diverse Scores empfohlen. ESC und EAS favorisieren das SCORE-System, das sich auf umfangreiche, in europäischen Populationen erhobene Daten stützt. Mithilfe der SCORE-Charts lässt

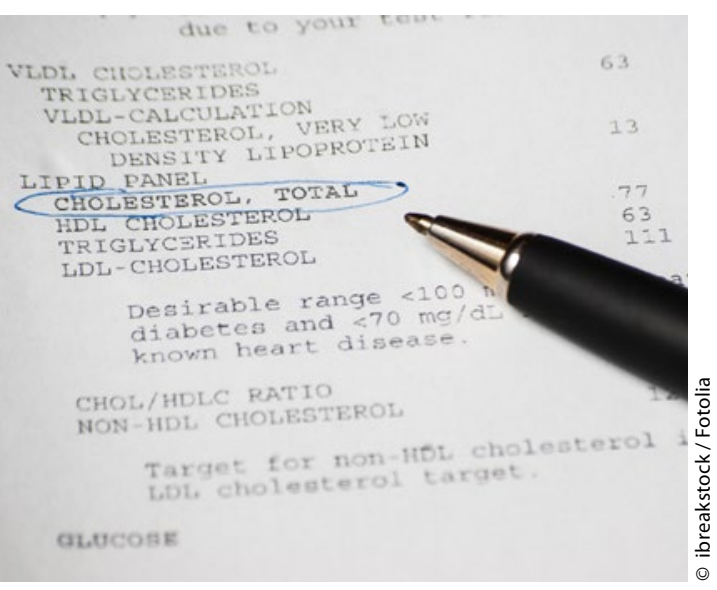

Patienten mit "hohem Risiko" sollten einen LDL-Cholesterinspiegel $<100 \mathrm{mg} / \mathrm{dl}$ $(<2,6 \mathrm{mmol} / \mathrm{l})$ erreichen. sich das 10-Jahres-Risiko für ein tödliches kardiovaskuläres Ereignis ermitteln. Eine solche Risikobestimmung wird bei asymptomatischen Erwachsenen im Alter über 40 Jahre ohne kardiovaskuläre Erkrankung, Diabetes, Nierenerkrankung oder familiäre Hypercholesterinämie (FH) empfohlen.

Bei Patienten mit Herzerkrankungen, bei Diabetikern und jenen mit bereits vorliegenden Zielorganschäden sowie bei individueller Häufung mehrerer Risikofaktoren wie Hypertonie und Dyslipidämie erübrigt sich die SCORE-Bestimmung, da diese Personen automatisch unter die Kategorie „sehr hohes kardiovaskuläres Risiko" fallen.

Dies gilt ebenso für Patienten mit Nierenerkrankungen. Hier wurde jedoch eine leichte Modifizierung vorgenommen. Galt in bisherigen ESC-Leitlinien eine GFR $<60 \mathrm{ml} / \mathrm{min}$ als Kriterium für ein „sehr hohes kardiovaskuläres Risiko", wird die Grenze nun bei $<30 \mathrm{ml} /$ min gezogen. Für Patienten mit einer GFR von $30-59 \mathrm{ml} / \mathrm{min}$ gilt nun die Kategorie „hohes kardiovaskuläres Risiko“.

\section{Zielwerte-Konzept beibehalten}

Primärer Ansatzpunkt der Lipidtherapie ist und bleibt das LDL-Cholesterin. Je höher das individuelle kardiovaskuläre Risiko, desto niedriger ist der für die LDL-Senkung vorgegebene Zielwert. Dass dieses Konzept nicht „evidenzbasiert" ist, ist auch den Leitlinien-Autoren klar. Randomisierte Studien, in denen die klinischen Auswirkungen einer therapeutischen Einstellung auf unterschiedliche LDL-Zielwerte untersucht wurden, gibt es nicht. Dennoch will man in Europa - anders als in den USA - aus pragmatischen Gründen das Konzept der risikobasierten LDL-Zielwerte, die der Orientierung für Ärzte und Patienten dienen sollen, beibehalten.
Bei Patienten der Kategorie „sehr hohes Risiko" wird ein LDL-Zielwert $<70$ $\mathrm{mg} / \mathrm{dl}(1,8 \mathrm{mmol} / \mathrm{l})$ empfohlen und/oder eine LDL-Reduktion um mindestens $50 \%$, wenn der Ausgangswert im Bereich zwischen $70 \mathrm{mg} / \mathrm{dl}$ und $135 \mathrm{mg} / \mathrm{dl}$ $(1,8 \mathrm{mmol} / \mathrm{l}$ und $3,5 \mathrm{mmol} / \mathrm{l})$ liegt (Klasse-I/B- statt bisher -I/A-Empfehlung).

Bei Patienten der Kategorie „hohes Risiko" wird weiterhin empfohlen, LDL-CSpiegel $<100 \mathrm{mg} / \mathrm{dl}(<2,6 \mathrm{mmol} / \mathrm{l})$ anzustreben. Hinzugekommen ist jetzt die Empfehlung, alternativ das LDL-Cholesterin um mindestens $50 \% \mathrm{zu}$ senken, wenn der Ausgangswert im Bereich zwischen $100 \mathrm{mg} / \mathrm{dl}$ und $200 \mathrm{mg} / \mathrm{dl}(2,6-5,1$ $\mathrm{mmol} / \mathrm{l}$ ) liegt (Klasse I/B).

Für Patienten mit niedrigem oder nur moderat erhöhtem Risiko wird nach wie vor ein Zielwert von $<115 \mathrm{mg} / \mathrm{dl}(<3$ $\mathrm{mmol} / \mathrm{l}$ ) vorgegeben (Klasse IIa/C).

\section{Kombinationen von Lipidsenkern}

Wenn klar ist, welcher Zielwert aufgrund des individuellen Risikos maßgeblich ist, sollte der Arzt die prozentuale LDL-Senkung berechnen, die nötig ist, um diesen Wert zu erreichen. Dann sollte ein Statin in einer Dosis, die eine solche LDL-Reduktion ermöglichen könnte, gewählt werden. Da Patienten unterschiedlich auf Statine ansprechen, kann eine Auftitrierung nötig sein.

Wird mit der höchsten noch verträglichen Statindosis der LDL-Zielwert nicht erreicht, ist an Lipidsenker-Kombinationen zu denken. Am ehesten sollte gemäß den neuen Leitlinien eine Kombination mit dem Cholesterinresorptionshemmer Ezetimib in Betracht gezogen werden (jetzt Klasse IIa/B statt zuvor IIb/B). Auch eine Kombination mit einem Gallensäure bindenden Ionenaustauscher kann man erwägen (Klasse IIb/B).

Erstmals haben die PCSK9-Hemmer Eingang in die Lipidleitlinien gefunden. Die neuen Cholesterinsenker können in Betracht gezogen werden bei Patienten der Kategorie „sehr hohes Risiko“, die trotz ausgereizter Therapie mit einem Statin in Kombination mit Ezetimib weiterhin anhaltend hohe LDL-Werte aufweisen (Klasse IIb/C). Peter Overbeck

ESC-Kongress, 27.-31.08.2016, Rom. 2016 ESC/EAS Guidelines for the Management of Dyslipidaemias, Eur Heart J 2016 